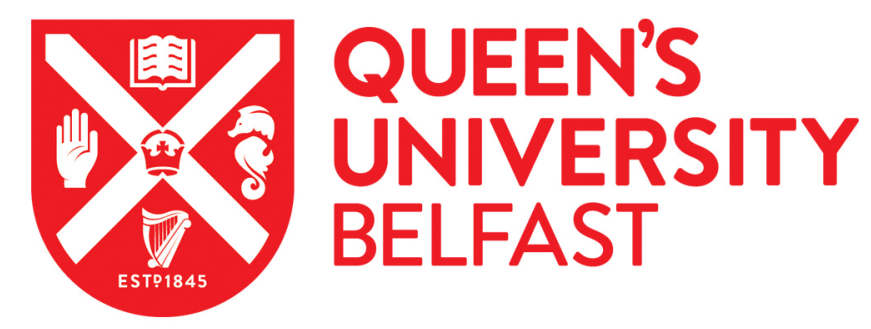

\title{
Frequency Diverse Array OFDM Transmitter for Secure Wireless Communication
}

Ding, Y., Zhang, J., \& Fusco, V. (2015). Frequency Diverse Array OFDM Transmitter for Secure Wireless Communication. Electronics Letters, 51(17), 1374-1376. https://doi.org/10.1049/el.2015.1491

\author{
Published in: \\ Electronics Letters
}

Document Version:

Peer reviewed version

Queen's University Belfast - Research Portal:

Link to publication record in Queen's University Belfast Research Portal

Publisher rights

(C) Copyright 2015 IEEE

This paper is a postprint of a paper accepted by Electronics Letters and is subject to Institution of Engineering and Technology Copyright. The copy of record will be available at IET Digital Library.

\section{General rights}

Copyright for the publications made accessible via the Queen's University Belfast Research Portal is retained by the author(s) and / or other copyright owners and it is a condition of accessing these publications that users recognise and abide by the legal requirements associated with these rights.

Take down policy

The Research Portal is Queen's institutional repository that provides access to Queen's research output. Every effort has been made to ensure that content in the Research Portal does not infringe any person's rights, or applicable UK laws. If you discover content in the Research Portal that you believe breaches copyright or violates any law, please contact openaccess@qub.ac.uk. 


\section{Frequency Diverse Array OFDM Transmitter for Secure Wireless Communication}

\author{
Y. Ding, J. Zhang and V. Fusco
}

\begin{abstract}
In this paper the frequency diverse array (FDA) concept is employed to construct an orthogonal frequency-division multiplexing (OFDM) transmitter that has the capability of securing wireless communication in free space directly in the physical-layer without the need for mathematical encryption. The characteristics of the proposed scheme in terms of its secrecy performance, is validated via bit error rate (BER) simulation under both high and low signal to noise ratio (SNR) scenarios using the IEEE 802.11 OFDM physical-layer specification.
\end{abstract}

Introduction: The frequency diverse array (FDA) technique has been developed in recent years by the radar community [1]-[8]. Unlike in conventional phased transmitter arrays where the signals applied onto each array radiating element are identically modulated with a constant radio frequency (RF) carrier subject to amplitude and phase weightings, the FDA approach employs a small amount of carrier frequency offset across the array elements. This offset enables automatically steered beams, and/or range-dependent radiation patterns, i.e., properties that are desirable for radar applications.

The FDA concept was first introduced in [1]. Here the range-dependent property of far-field beam patterns formed using the method was pointed out. In [2], [3] it was shown that the beam patterns were time-dependent. Mathematical analysis of the FDA far-field beam pattern properties was developed in [4]-[6], while possible radar implementation architectures were described in [7], [8].

This paper, to the authors' knowledge, is the first time that; a) the link between FDA and orthogonal frequency-division multiplexing (OFDM) has been established, $\mathrm{b}$ ) the FDA OFDM transmitters have been proposed and used for the purpose of secure wireless communication.

Frequency Diverse Array (FDA) OFDM Transmitters: Consider an $\mathrm{N}$ by-1 FDA with uniform array element spacing $d$ and uniform frequency increment $\Delta f$ across the array aperture, the signal $S$ received at a location $\left(R_{1}, \theta\right)$ in free space can be expressed as

$$
\boldsymbol{S}=\sum_{n=1}^{N}\left(\boldsymbol{A}_{n} \cdot e^{-j\left[2 \pi f_{n}\left(t-R_{n} / c\right)-\boldsymbol{\phi}_{n}\right]}\right)
$$

where $f_{n}$ is the RF carrier frequency of the signal applied to the $n^{\text {th }}$ array element, $f_{n}=f_{1}+(n-1) \Delta f$. $\boldsymbol{A}_{n}$ is a complex number representing information data for transmission.

$$
R_{n}=R_{1}-(n-1) d \cos \theta
$$

is the receiver displacement relative to the $n^{\text {th }}$ array element. The first element is set as reference. $\theta$ is the azimuth angle with boresight along $90^{\circ}$, and $c$ denotes the speed of light. Phase $\phi_{n}$ is the initial phase of the $n^{\text {th }} \mathrm{RF}$ carrier. Assuming that the receiver node is located in the far-field region, then the path loss from each array element is approximately identical, and is therefore omitted in (1).

Rewriting (1) as (3),

$$
\boldsymbol{S}=\sum_{n=1}^{N}\left(\boldsymbol{A}_{n} \cdot e^{j \phi_{n}} \cdot e^{j 2 \pi f_{n} R_{n} / c} \cdot e^{-j 2 \pi f_{1} t} \cdot e^{-j 2 \pi(n-1) \Delta f t}\right)
$$

In order to allow information $\boldsymbol{A}_{n}$ to be extracted from $\boldsymbol{S}$, the $N$ summation terms have to be separated and the four exponential coefficients removed. This is performed as follows.

At the receiver side frequency down-conversion by multiplying by a RF carrier $e^{j 2 \pi f_{1} t}$ eliminates the coefficient $e^{-j 2 \pi f_{1} t}$ in (3). The separation of the $N$ summation terms and the removal of $e^{-j 2 \pi(n-1) \Delta f t}$ can be concurrently achieved with the help of the orthogonality property of the function sets $e^{-j p x}$ for different integer $p$ within any $2 i \pi$ range. Namely,

$$
\frac{1}{2 i \pi} \int_{2 \pi a}^{2 \pi(a+i)} e^{-j p x} e^{j q x} d x= \begin{cases}0 & (\forall p, q \in Z, \forall i \in N, p \neq q) \\ 1 & (\forall p, q \in Z, \forall i \in N, p=q)\end{cases}
$$

As a consequence by comparison the time $t$ in (3) has to be confined within a range between $a / \Delta f$ and $(a+i) / \Delta f, a$ can be any positive real number. In other words the symbol duration $\Delta T$ is required to take values of $i / \Delta f$ in order for data recovery to occur. Fig. 1 depicts the frequency spectrum of each carrier in signal $S$ within $\Delta T$ for $i=1$ and $N=5$. This is calculated by performing a Fourier transform after multiplying (3) by the rectangular window function $\operatorname{rect}(t)$, defined in (5),

$$
\operatorname{rect}(t)=\left\{\begin{array}{rr}
1 & t \in[a / \Delta f,(a+1) / \Delta f] \\
0 & \text { others }
\end{array}\right.
$$

Here we have assumed that $\boldsymbol{A}_{n}$ for each $n$ have the same absolute value $|\boldsymbol{A}|$. It is observed that the carriers are orthogonal to each other in frequency domain at their respective centre frequency. Inspection of the signal spectrum in Fig. 1 shows that by confining the symbol time period to $1 / \Delta f$ the detected signals are OFDM modulated signals with each subcarrier being transmitted separately via each FDA element.

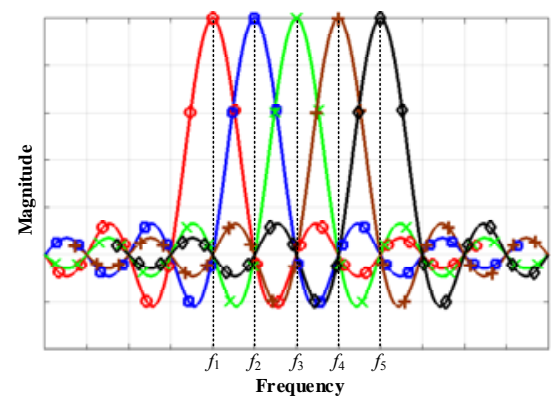

Fig. 1 Frequency spectrum of each carrier in received signal $\mathbf{S}$ in (3) upon confining the symbol time period $\Delta T$ as $1 / \Delta f, N=5$ for this example.

FDA OFDM Transmitter for Secure Wireless Communication: In conventional OFDM systems each modulated symbol weighted subcarrier is combined before being routed to the antenna array for transmission. For this scenario (6) gives the detected signals $\boldsymbol{S}^{\prime}$ along direction $\theta$ in free space,

$$
\boldsymbol{S}^{\prime}=\boldsymbol{A} \boldsymbol{F}(\theta) \cdot \sum_{n=1}^{N}\left(\boldsymbol{A}_{n} \cdot e^{-j 2 \pi f_{1} t} \cdot e^{-j 2 \pi(n-1) \Delta f t}\right)
$$

From (6) it can be concluded that the modulated OFDM signal waveforms are preserved along all $\theta$ with the ability to recover the transmitted information being determined by signal to noise ratios (SNRs) governed by the array factor $\boldsymbol{A F}(\theta)$, distance $R$ from the transmitter, and channel noise.

This characteristic, however, does not hold for the proposed FDA OFDM transmitter. By comparing (3) and (6) it can be seen that the FDA OFDM transmitter permits the possibility for manipulation of the phase of each sub-carrier in the OFDM signals by choosing the sub-carrier initial phase $\phi_{n}$ and selecting which array elements radiate each subcarrier, i.e., $\phi_{n}$ and $2 \pi f_{n} R_{n} / c$ in (3). Thus this provides an extra degree of freedom unavailable to the conventional OFDM transmitters. The result of this is the projection of different signal waveforms along different directions since $R_{n}$ is a function of $\theta$, (2).

The ability for signal waveform manipulation that the FDA OFDM transmitter possesses can be exploited by setting the summation of $\phi_{n}$ and $2 \pi f_{n} R_{n} / c$ be a constant $\Phi$ for each $n$ along an a-priori selected communication direction $\theta_{0}$ for the purpose of securing a wireless communication along that direction. $\Phi$ can be any real number, and it acts to describe the rotation of symbol constellation diagram in IQ space for each sub-carrier. This rotation can be fully compensated, only, by receivers located along $\theta_{0} . f_{n}$ is fixed for the $n^{\text {th }}$ sub-carrier. $R_{n}$ is a function of both $n$ and communication direction $\theta$, (2). Select $R_{n}$ according to (2) for the specified array element and given direction $\theta_{0}$ and solve for $\phi_{n}$. Under this condition

$$
\begin{aligned}
\boldsymbol{S}\left(\theta_{0}\right) & =\sum_{n=1}^{N}\left(\boldsymbol{A}_{n} \cdot e^{j \phi_{n}} \cdot e^{j 2 \pi f_{n} R_{n} / c} \cdot e^{-j 2 \pi f_{1} t} \cdot e^{-j 2 \pi(n-1) \Delta t}\right) \\
& =e^{j \Phi} \cdot \sum_{n=1}^{N}\left(\boldsymbol{A}_{n} \cdot e^{-j 2 \pi f_{1} t} \cdot e^{-j 2 \pi(n-1) \Delta f t}\right)=\left[e^{j \Phi} / \boldsymbol{A} \boldsymbol{F}\left(\theta_{0}\right)\right] \cdot \boldsymbol{S}^{\prime}\left(\theta_{0}\right)
\end{aligned}
$$


which indicates that the OFDM signal waveforms are well preserved along $\theta_{0}$. On the other hand, signal waveforms are scrambled at all directions other than $\theta_{0}$ since the summations of $\phi_{n}$ and $2 \pi f_{n} R_{n} / c$ are no longer a constant for each $n$ and the OFDM time waveform signature becomes distorted.

Simulation Results: In order to validate the superiority of the proposed FDA OFDM transmitter types over the conventional OFDM transmit architecture, the bit error rate (BER) spatial distributions in free space in the different systems are simulated under the following prerequisites:

- In IEEE 802.11 OFDM systems [9], used here to facilitate further discussion in this paper, block type training sequences are adopted where each sub-carrier is appended with an identical training preamble, consisting of both short and long training symbols. The preamble is utilized to determine, for each separate OFDM subcarrier, where the information data begins and what the phase reference of each sub-carrier is, i.e., $\phi_{n}+2 \pi f_{n} R_{n} / c$ along all directions if the mappings between array elements and sub-carriers are fixed in one data packet. As a consequence, in order to exert uncorrected distortion at all spatial directions other than $\theta_{0}$, the mappings need to be updated dynamically on a per transmitted symbol basis while satisfying $\phi_{n}+2 \pi f_{n} R_{n} / c=\Phi$ along desired $\theta_{0}$. This scheme not only disconnects the training sequences and their corresponding subcarrier data, but also scrambles the training preambles themselves, leaving little chance of eavesdropper data interception along directions other than that pre-specified.

- IEEE 802.11 OFDM (20 MHz channel spacing $B$ ) physical-layer specification [9] is adopted. No data coding and interleaving are performed. The OFDM signals contain 64 subcarriers, of which 52, 48 for data and 4 for pilot, are used for transmission. $f_{1} \approx 2.404 \mathrm{GHz}$ (channel 1 at $2.4 \mathrm{GHz}$ band), and $\Delta f=B / 64=312.5 \mathrm{kHz}$.

- A 9-by-1 isotropic antenna element array with half-wavelength spacing with respect to $f_{1}$ is used. Since $N=9<52$, it is inevitable that several sub-carriers are mapped onto a same array element for transmission. The mapping is performed by random assignment, i.e., for this example each transmitted OFDM symbol each of the 52 subcarriers has a $1 / 9$ chance to be assigned to each of the 9 array elements. This arrangement, compared with the conventional OFDM systems wherein all sub-carriers are combined, reduces the peak-toaverage power ratio (PAPR) of signals at the transmitter side, which leads to increased power efficiency of the transmit power amplifiers.

- Each OFDM packet consisting of around 800 random data bits is appended with standard IEEE 802.11 OFDM preambles. $10^{4}$ packets are used for each BER simulation, which allows BER down to $10^{-5}$ to be calculated.

- For fair comparison in terms of secrecy performance the signal to noise ratios (SNRs) detected by receivers located along pre-selected directions in both systems are normalized to be identical. AWGN contribution is identical in all directions. It is also assumed that legitimate receivers and eavesdropper receivers are located at a same distance away from the transmitter, (the case of different distances is equivalent to different receive SNRs).
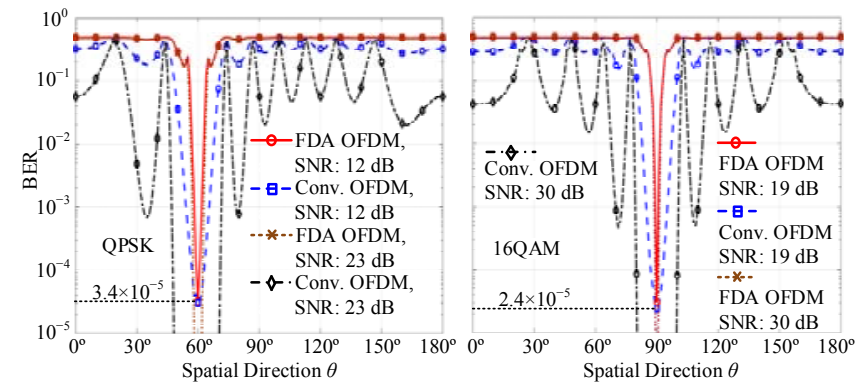

Fig.2 Simulated BER distributions in both conventional and FDA OFDM systems in free space. SNRs along selected communication direction $60^{\circ}$ or $90^{\circ}$ are set to $12 \mathrm{~dB}, 23 \mathrm{~dB}$ for $Q P S K$ case, and $19 \mathrm{~dB}, 30 \mathrm{~dB}$ for 16QAM case.

In Fig. 2 the BER simulation results obtained for $60^{\circ}$ and $90^{\circ}$ pre- defined communication directions respectively are depicted, other directions could have equally been selected. Both low and high SNR values are chosen for each case to facilitate BER main beam and sidelobe comparison in FDA and conventional OFDM systems. The sample results in Fig. 2 validate that the OFDM signal waveforms are well preserved only along pre-selected directions, i.e., in the example shown here for Gray-coded QPSK and 16QAM modulations respectively BERs detected along the directions of $60^{\circ}$ or $90^{\circ}$ in both conventional and FDA OFDM systems are approximately the same and follow the BER-SNR relationships stated in [10]. We can also conclude that FDA OFDM systems are able to greatly confine BER spatial spread around the selected communication directions and suppress BER sidelobes, especially under high SNR scenarios. This secrecy enhancement is off course obtained at the cost of radiating more energy into space since the system provides no beam-forming gain, see (7). This can be partially alleviated by only applying the FDA OFDM technique onto the appended training preamble in each data packet, and enabling beam-forming functionality for the real transmitted information data, i.e., hybridizing FDA and conventional OFDM transmitters.

Conclusion: An FDA inspired array-level OFDM transmitter was proposed that has the ability to secure wireless communication in free space at the physical-layer along a prescribed direction. The approach was validated via BER simulations using the IEEE 802.11 OFDM physical-layer specification. Experimental verification of the system suggested here will be the subject of later work. The FDA OFDM transmitter concept introduced in this paper should be useful in applications where enhanced security through physical-layer augmentation would add operational benefit.

Y. Ding, J. Zhang and V. Fusco (The ECIT Institute, Queen's University of Belfast, Belfast, BT3 9DT, UK, yding03@qub.ac.uk)

\section{References}

1. Antonik P., Wicks, M., Griffiths, H., Baker, C.: 'Frequency diverse array radars', IEEE Radar Conf., Verona, NY, USA, April 2006, pp. 215-217, doi: 10.1109/RADAR.2006.1631800

2. Secmen, M., Demir, S., Hizal, A., Eker, T.: 'Frequency diverse array antenna with periodic time modulated pattern in range and angle', IEEE Radar Conf., Boston, MA, USA, April 2007, pp. 427-430, doi: 10.1109/RADAR.2007.374254

3. Huang, J.: 'Frequency diversity array: theory and design', Ph.D. dissertation, Dept. Electronic and Electrical Eng., University College London, London, U.K., 2010.

4. Sammartino, P., Baker, C., Griffiths, H.: 'Range-angle dependent waveform', IEEE Radar Conf., Washington DC, USA, May 2010, pp. 511-515, doi: 10.1109/RADAR.2010.5494568

5. Wang, W., Shao, H., Cai, J.: 'Range-angle-dependent beamforming by frequency diverse array antenna', Int. J. Antennas Propag., 2012, pp. 110, doi: $10.1155 / 2012 / 760489$

6. Wang, W.: 'Range-angle dependent transmit beam pattern synthesis for linear frequency diverse arrays', IEEE Trans. Antennas Propag., 2013, 61, (8), pp. 4073-4081, doi: 10.1109/TAP.2013.2260515

7. Wicks, M., Antonik, P.: 'Frequency diverse array with independent modulation of frequency, amplitude, and phase', U.S. patent 7,319,427, Jan. 15, 2008.

8. Jones A., Rigling, B,: 'Planar frequency diverse array receiver architecture', IEEE Radar Conf., Atlanta, USA, May 2012, pp. 145-150, doi: 10.1109/RADAR.2012.6212127

9. Wireless LAN Medium Access Control (MAC) and Physical Layer (PHY) Specification, IEEE Std. 802.11, 2012.

10. Shafik, R., Rahman, S., Islam, R.: 'On the extended relationships among EVM, BER and SNR as performance metrics', Int. Conf. Elect. Comput. Eng., 2006, pp. 408-411, doi: 10.1109/ICECE.2006.355657 